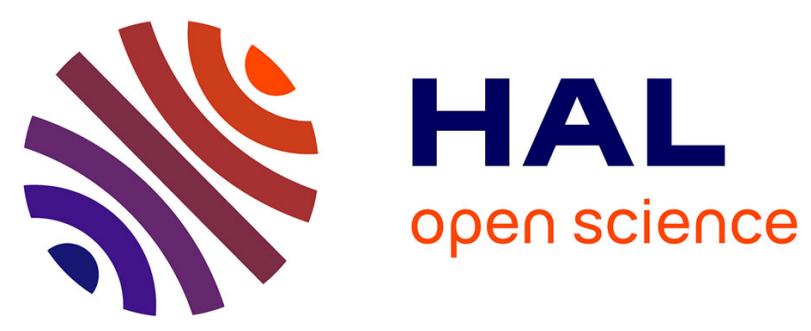

\title{
Chi-squared Distribution Approximation for Probabilistic Energy Equalizer Implementation in Impulse-Radio UWB Receiver
}

Sami Mekki, Jean-Luc Danger, Benoit Miscopein, Joseph J. Boutros

\section{- To cite this version:}

Sami Mekki, Jean-Luc Danger, Benoit Miscopein, Joseph J. Boutros. Chi-squared Distribution Approximation for Probabilistic Energy Equalizer Implementation in Impulse-Radio UWB Receiver. 2008. hal-00299912

\section{HAL Id: hal-00299912 https://hal.science/hal-00299912}

Preprint submitted on 17 Jul 2008

HAL is a multi-disciplinary open access archive for the deposit and dissemination of scientific research documents, whether they are published or not. The documents may come from teaching and research institutions in France or abroad, or from public or private research centers.
L'archive ouverte pluridisciplinaire HAL, est destinée au dépôt et à la diffusion de documents scientifiques de niveau recherche, publiés ou non, émanant des établissements d'enseignement et de recherche français ou étrangers, des laboratoires publics ou privés. 


\title{
Chi-squared Distribution Approximation for Probabilistic Energy Equalizer Implementation in Impulse-Radio UWB Receiver
}

\author{
Sami Mekki ${ }^{1}$, Jean-Luc Danger ${ }^{1}$, Benoit Miscopein ${ }^{2}$ and Joseph J. Boutros ${ }^{3}$ \\ ${ }^{1}$ École Nationale Supérieure des Télécommunications, 46 rue Barrault, 75634 Cédex 13, Paris, France. \\ ${ }^{2}$ France Télécom R\&D, 28 Chemin du vieux chêne, 38243 Meylan Cédex, France. \\ ${ }^{3}$ Texas A\&M University at Qatar, Education City, PO Box 23874, Doha, Qatar. \\ Email: \{mekki, danger\}@enst.fr, benoit.miscopein@orange-ftgroup.com, boutros@tamu.edu
}

\begin{abstract}
This paper addresses the hardware implementation feasibility assessment of a probabilistic energy equalizer for UWB impulse radio (IR) transmission. The receiver is based on energy detection and targets data rates greater than $100 \mathrm{Mbps}$. The aim of this study is to bypass certain complex mathematical functions like the chi-squared $\left(\chi^{2}\right)$ distribution and approximate it by functions easier to implement. Fixed point data types simulations are achieved and compared to the results obtained from the reference floating point non-approximated function. Simulation results, under IEEE 802.15.3a channel models, confirm our choice which allows an easy hardware implementation for these complex functions.
\end{abstract}

\section{INTRODUCTION}

Ultra-wideband impulse radio (IR) is considered as a promising candidate for indoor communications and wireless sensor networks, as described in [1]. Both transmitter and receiver are facing difficulties of implementation due to the technological limits. Coherent IR UWB reception based on Rake receiver is limited in number of implementable Rake fingers [2]. An alternative is given by the transmitter reference (TR) method [3], however the electronic architecture is more complex as it needs analog delay lines and mixers. Non-coherent energy detection receiver is far less complex as a few components like shottky diodes and capacitors suffice. Nevertheless transmitting impulses at high data rate causes inter-symbol interference [4], [5], [6] which could seriously decrease the performances of a simple energy detector.

In [7], it was proposed a probabilistic energy equalizer that handles the interferences among symbols and inside the symbols in an $M$ - array pulse position modulation (M-PPM). However, equalization function is mathematically complex and leads to hardly feasible implementations. The problem comes mainly from the energy expression, which follows a chi-squared $\left(\chi^{2}\right)$ distribution [8]. Several papers have looked for an approximation of the chi-squared distribution [9], [10]. Nevertheless the proposed approximations remain complex for digital implementations.

In this paper, the approximation defined in [11] is studied for a fixed point data types implementation. The chisquared approximation is implemented and tested within the probabilistic energy detector equalizer proposed in [7]. The equalizer is followed by a channel decoder which allows joint channel decoding in the same iterative loop.

This article is organized as follows:

The system model is defined in Section II where the conditional distribution of the received energy is established for equalization purpose. Section III reviews the energy equalization principle. In Section IV, the conditioned energy distribution defined in Section II is approximated by a simple function for hardware implementation. Results with the approximated distribution are compared to the chisquared distribution in Section V. In the same section the hardware implementation results in fixed point precision data types are also depicted and compared to the theoretical results in floating point precision. Final remarks, conclusion and discussion about forthcoming work in the field are given in Section (VI).

\section{System Model}

We consider an UWB communication system based on impulse radio (IR) transmission with a signal bandwidth $W$. An $M-$ ary Pulse Position Modulation (M-PPM) is assumed for symbol modulation, where each symbol is defined by the position of a pulse over a time symbol $T_{s}$. However, transmitting pulses at high data rate over dispersive channels [12] leads to inter-symbol interference (ISI) or inter-slot interference (IStI). It means that the channel impulse response of a transmitted pulse may provoke collisions on the following slots or the following symbols.

The resulting received signal in the time interval $\left[(n-1) T_{s}, n T_{s}[\right.$ is defined as

$$
\mathbf{y}_{n}(t)=\mathbf{s}_{n}(t)+z_{n}(t)=\sum_{k=0}^{\infty} x_{n-k}(t)+z_{n}(t)
$$

where $z_{n}(t)$ is an additive white Gaussian noise with mean zero and variance $\sigma^{2}$ and $x_{n-k}(t)$ being the channel 
response of the $(n-k)^{t h}$ transmitted symbol defined by:

$$
x_{n-k}(t)=p\left(t-A_{n-k} T_{\text {slot }}\right) \otimes h(t)
$$

where $h(t)$ is the channel impulse response, $\otimes$ denotes the convolution product, $p(t)$ is the pulse shape, $T_{\text {slot }}$ is the time slot duration for an M-PPM modulation and $A_{n, k}$ takes value in $\{0,1, \ldots, M-1\}$ according to transmitted symbol.

Without loss of generality, the pulse is considered as being a Dirac $\delta(t)$ function, thus $x_{n-k}(t)=h\left(t-A_{n-k} T_{\text {slot }}\right)$. This assumption does not affect our logical thinking.

It should be noted that the receiver can handle only a finite number of interfering symbols. Let $K$ be the number of interfering symbols considered by the receiver. So, it assumes that the UWB received pulse can not exceed $P$ interfering slots linked to $K$ by $P=(K-1) M+1$. The actual value of the number of interfering symbols might be larger than the supposed value $K$. Thus, $s_{n}(t)$ turns to

$$
\mathbf{s}_{n}(t)=\sum_{0}^{K-1} x_{n-k}(t)
$$

The radio frequency $(\mathrm{RF})$ reception architecture is assumed to be an energy detector receiver so that the energy per time slot $m$ in $n^{t h}$ symbol is given by

$$
\mathcal{E}_{n, m}=\int_{n T_{s}+(m-1) T_{\text {slot }}}^{n T_{s}+(m) T_{\text {slot }}}\left(\mathbf{s}_{n}(t)+z_{n}(t)\right)^{2} d t
$$

where $T_{s}=M T_{\text {slot }}$. For digital processing concerns, (4) must be discretized into a sum of finite samples. In [13], it was shown that the energy of a signal, of duration $T_{\text {slot }}$, with a bandwidth $W$ can be approximated by the sum of $2 W T_{\text {slot }}$ samples. This leads to

$$
\mathcal{E}_{n, m}=\sum_{\ell=1}^{2 L}\left(s_{n, m}^{\ell}+z_{n, m}^{\ell}\right)^{2}
$$

where $2 L=2 W T_{\text {slot }}$ is the number of degrees of freedom (DoF) over the interval $T_{\text {slot }}$, and $s_{n, m}^{\ell}$ and $z_{n, m}^{\ell}$ are respectively the $\ell^{t h}$ sample of $\mathbf{s}_{n}(t)$ and $z_{n}(t)$ in $m^{\text {th }}$ slot of $n^{\text {th }}$ symbol.

The received energy per slot defined by $\mathcal{E}_{n, m}=$ $\sum_{\ell=1}^{2 L} \mathcal{X}_{\ell}^{2}$, where $\mathcal{X}_{\ell}=s_{n, m}^{\ell}+z_{n, m}^{\ell}$, follows a central chisquared [8] distribution with $2 L \operatorname{DoF}$ if $\sum_{\ell}\left(s_{n, m}^{\ell}\right)^{2}=0$, given by

$$
p\left(\mathcal{E}_{n, m} \mid 0\right)=\frac{1}{\sigma^{2 L} 2^{L} \Gamma(L)} \mathcal{E}_{n, m}^{L-1} e^{\frac{-\mathcal{E}_{n, m}}{2 \sigma^{2}}}
$$

where $\Gamma(z)$ is the gamma function [14]. Furthermore, if $\sum_{\ell}\left(s_{n, m}^{\ell}\right)^{2} \neq 0$, the energy per slot follows a non-central chi-squared distribution [8] with $2 L$ DoF given by

$$
\begin{aligned}
p\left(\mathcal{E}_{n, m} \mid B_{n, m}\right)= & \frac{1}{2 \sigma^{2}}\left(\frac{\mathcal{E}_{n, m}}{B_{n, m}}\right)^{\frac{L-1}{2}} e^{-\frac{\left(\mathcal{E}_{n, m}+B_{n, m}\right)}{2 \sigma^{2}}} \\
& I_{L-1}\left(\frac{\sqrt{B_{n, m} \mathcal{E}_{n, m}}}{\sigma^{2}}\right)
\end{aligned}
$$

where the non-centrality parameter

$$
B_{n, m} \triangleq \sum_{\ell=1}^{2 L}\left(s_{n, m}^{\ell}\right)^{2}=\left(\mathbf{s}_{n, m}\right)^{2}
$$

is the resulting energy on the slot $m$ of the $n^{\text {th }}$ symbol without considering the additive noise. $I_{L-1}(u)$ is the $(L-$ $1)^{t h}$-order modified Bessel function of the first kind [14].

\section{Energy Equalization Principle}

To benefit from the iterative process of a communication system, we consider a probabilistic equalizer that can be embedded into the iterative loop of a channel decoder based on SISO (Soft-Input/Soft-Output) decoding.

Thus, the considered equalizer takes the accumulated energy per slot (i.e. $\mathcal{E}_{n, m}$ ) and per symbol (i.e. $\mathscr{E}_{n}=$ $\left.\left(\mathcal{E}_{n, 1}, \mathcal{E}_{n, 2}, \ldots, \mathcal{E}_{n, M}\right)\right)$ as reference, in order to retrieve the transmitted symbol $x_{n}$. So the detector computes a conditioned probability $p\left(\mathscr{E}_{n} \mid x_{n}\right)$ regarding the interfering

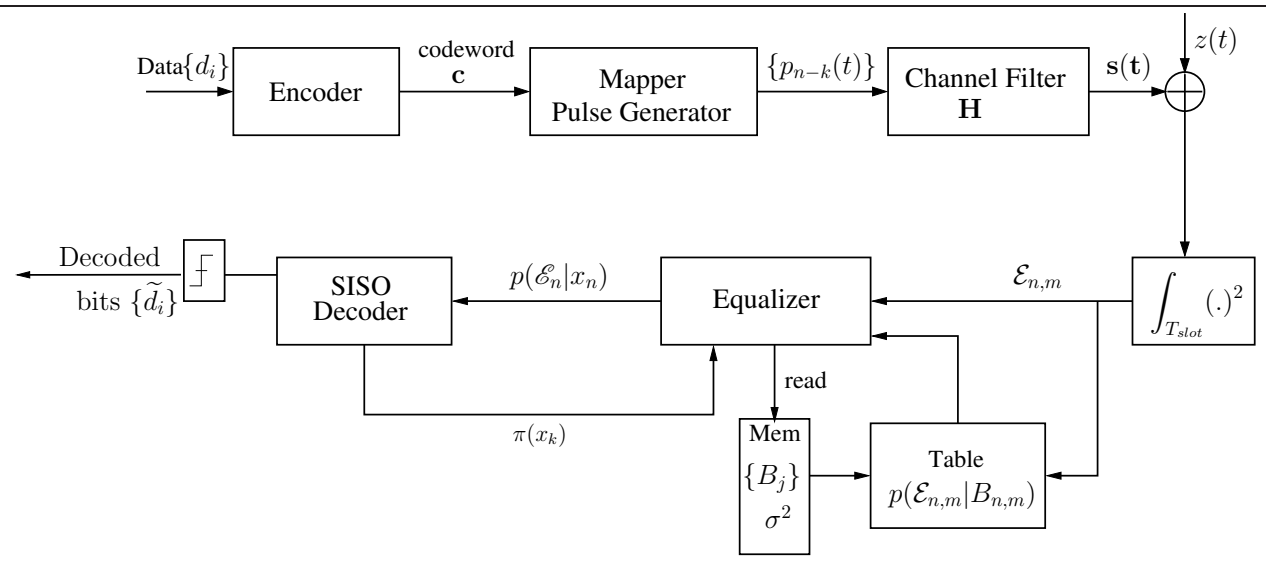

Fig. 1. Transmitter and receiver design. 
symbols on $x_{n}$. It has been shown in [7] that the equalization is performed by computing

$$
\begin{aligned}
& p\left(\mathscr{E}_{n} \mid x_{n}\right)= \\
& \sum_{x_{n-1}} \ldots \sum_{x_{n-K+1}}\left(\prod_{m=1}^{M} p\left(\mathcal{E}_{n, m} \mid B_{n, m}\right) \prod_{k=1}^{K-1} \pi\left(x_{n-k}\right)\right)
\end{aligned}
$$

where $\pi\left(x_{n-k}\right)$ is the a priori probability provided by the SISO decoder and $p\left(\mathcal{E}_{n, m} \mid B_{n, m}\right)$ is defined in Section II. It was also established that the set of all the possible values that $B_{n, m}$ could take, has a finite cardinal. Figure 1 resumes the transmission and the receiver design under consideration.

In order to reduce the complexity and make the equalizer feasible, we investigate the implementation in finite precision.

Moreover the probability given by Equation (9) needs some mathematical simplifications and approximations of the probability density function (pdf) $p\left(\mathcal{E}_{n, m} \mid B_{n, m}\right)$, corresponding either to the central (6) or non-central chisquared (7) distribution. This will be investigated in the following section.

\section{Chi-Squared Distribution Approximation for HARDWARE IMPLEMENTATION}

The chi-squared distribution defined by (6) and (7) is a three variable function $\left(\mathcal{E}_{n, m}, B_{n, m}\right.$ and $\left.\sigma^{2}\right)$. Thus, building a look-up table according to these parameters would occupy a great memory. For instance, if the energy distribution is coded with 7 bits and if $\mathcal{E}_{n, m}, B_{n, m}$ and $\sigma^{2}$ are respectively 14 -bit, 6 -bit and 6 -bit long, the space memory allocated to this look-up table would occupy $448 \mathrm{Mbits}$ (or 56Mbytes). This corresponds to a costly silicon area in a FPGA or ASIC technology and thus incompatible with low-cost constraints.

An approximation for the chi-squared distribution is thus necessary. In the literature, there are some proposals for the calculation of the non-central chi-squared distribution [15] and the use of the normal approximation to the chi-squared distribution [9], [10], [11], but those approximations require high bit precision and are therefore too complex for digital design.

An intuitive approximation can be found by considering the Remark in [11] which stands that when a variable $\Sigma$ is used to approximate a variable $\Omega$, it is equivalent to match the mean and variance of $\Sigma$ and $\Omega$.

It is notably shown in [11], that a chi-squared distribution can be approximated by a Gaussian distribution. However the smaller the number of DoF $2 L$, the larger the approximation error. Due to the large bandwidth $W$ in UWB-IR, the number DoF could be big enough [16] to consider the Gaussian distribution as an approximation to the chi-squared density. For instance $2 L$ is around 30 for $W=3 \mathrm{GHz}$ and $T_{\text {slot }}=5 \mathrm{~ns}$. According to the previous Remark, the Gaussian approximation has the same mean and variance as the non-central chi-squared distribution, i.e. $\mathcal{E}_{n, m} \sim N\left(m_{\chi^{2}}, \sigma_{\chi^{2}}^{2}\right)$, given by [8]:

$$
\begin{aligned}
m_{\chi^{2}} & =2 L \sigma^{2}+B_{n, m} \\
\sigma_{\chi^{2}}^{2} & =4 L \sigma^{4}+4 \sigma^{2} B_{n, m}
\end{aligned}
$$

This can be extended to the central chi-squared distribution by considering $B_{n, m}=0$.

Using these results and the aforementioned assumptions, we obtain the approximation for the energy distribution (noticed $\tilde{p}$ ) per slot, $\forall B_{n, m} \geq 0$ and $2 L>>2$ as

$p\left(\mathcal{E}_{n, m} \mid B_{n, m}\right) \approx \widetilde{p}\left(\mathcal{E}_{n, m} \mid B_{n, m}\right)=\frac{\exp \left(-\frac{\left(\mathcal{E}_{n, m}-m_{\chi^{2}}\right)^{2}}{2 \sigma_{\chi^{2}}^{2}}\right)}{\sqrt{2 \pi \sigma_{\chi^{2}}^{2}}}$

Figure 2(a) shows the error measured by $\mid p\left(\mathcal{E}_{n, m} \mid B_{n, m}\right)-$ $\widetilde{p}\left(\mathcal{E}_{n, m} \mid B_{n, m}\right) \mid$ for $\mathcal{E}_{n, m} \geq 0, B_{n, m}>0$ and $\sigma^{2}=1$.

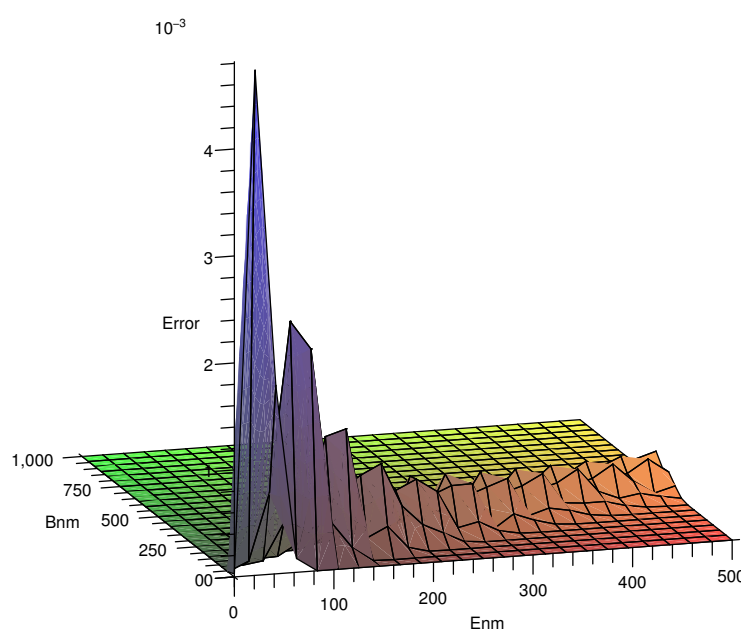

(a) Error for $\sigma^{2}=1$

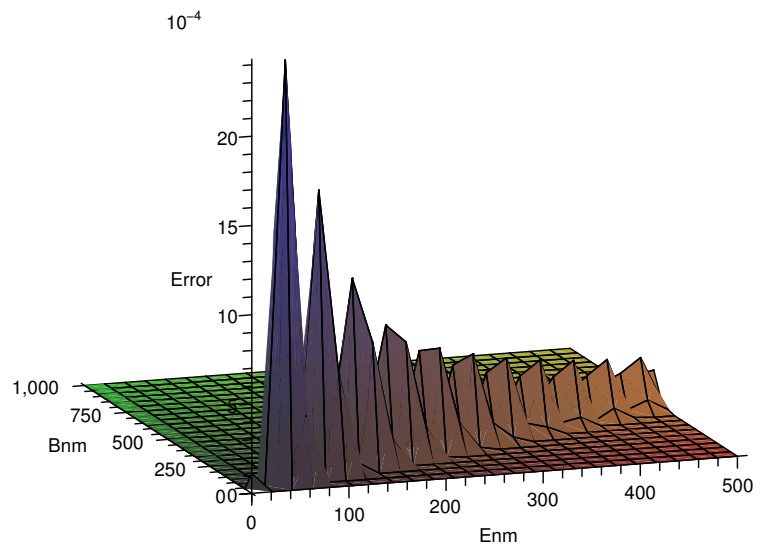

(b) Error for $\sigma^{2}=0.5$

Fig. 2. Error measured by $\left|p\left(\mathcal{E}_{n, m} \mid B_{n, m}\right)-\widetilde{p}\left(\mathcal{E}_{n, m} \mid B_{n, m}\right)\right|$ for $\forall \mathcal{E}_{n, m} \geq 0, B_{n, m}>0$ 
It is noticed that the error tends to zero as $\sigma^{2}$ decreases (Figure 2(b)). According to [7], the energy equalizer operates at $\sigma^{2}<1$; i.e. $\sigma^{2}=1$ corresponds to $S N R=-3 d B$ for a pulse energy equals to unity in coded system. So, the maximum error, considered between the chi-squared and Gaussian distributions, is $\epsilon=5 \times 10^{-3}$ as shown in Figure 2(a). We denote the normal function by

$$
\phi(t)=\frac{1}{\sqrt{2 \pi}} e^{-t^{2} / 2}
$$

Using (10), (11) and (13), equation (12) can be rewritten as follows

$$
\widetilde{p}\left(\mathcal{E}_{n, m} \mid B_{n, m}\right)=\frac{1}{\sqrt{\sigma_{\chi^{2}}^{2}}} \phi\left(\frac{\mathcal{E}_{n, m}-m_{\chi^{2}}}{\sqrt{\sigma_{\chi^{2}}^{2}}}\right)
$$

As the energy distribution is simply deduced from the normal function $\phi(t)$, the digital implementation can only use two look-up tables. The first one contains the values of the normal function $\phi(t), \forall t \geq 0$. The second one contains the values of the ratio $1 / \sqrt{x}, \forall x>0$. The input/output precision of the look-up tables will be analyzed in the simulation section according to the hardware constraints.

\section{Simulation Results}

The BER computation of the energy equalizer with the approximated Gaussian distribution has been performed via simulations in both floating point precision and fixed point precision data types. Before considering simulations in fixed point precision, we compare the performance of the receiver with the Gaussian approximation (12) and with the exact calculation of the chi-squared distribution in floating point precision.

The block fading multipath channel is generated randomly according to IEEE 802.15.3a UWB channel models CM1 and CM2 [12]. Channel estimation is out of the scope of this paper. The channel state information (CSI) is assumed perfectly known at the receiver side. Nevertheless, channel parameters can be approached by the mean of the expectation-maximization (EM) algorithm as studied in [17].

\section{A. Chi-squared versus Gaussian approximation simula- tions in double precision}

We consider an UWB-IR system as defined in Figure 1. Transmission is ensured by a $4-\mathrm{PPM}$ modulation at $100 \mathrm{Mbit} / \mathrm{s}$. Thus we get 2 bits per transmitted symbol. We have implemented a duo-binary turbo code as it is defined in the standards [18], [19]. This channel coder is chosen because it is suited to QPSK (quadratic phase shift keying) and 4-PPM modulations. The encoded data, at the input of the encoder, are 864-bit long blocks. The turbo encoder rate is $1 / 2$ and 10 iterations of the SISO decoder are performed at the receiver side. The equalizer is jointly implemented into the iterative loop of the decoder to benefit from the iterative process of the decoder. The efficiency of the energy equalizer will not be treated in this paper, the reader should refer to [7] for more details concerning the equalizer performances.

The receiver assumes that there are only two interfering symbols, i.e. $K=2$ and $P=5$, but the real number of interfering symbols could be more. The CSI is assumed over $P$ time slots duration and not otherwise. In our case, for a data rate of $100 \mathrm{Mbps}$, the time slot duration is $5 \mathrm{~ns}$, so the receiver has a perfect CSI only over $25 \mathrm{~ns}$. This duration is sufficient for channel models as CM1 and CM2, although their respective maximum excess delay are $80 \mathrm{~ns}$ and $115 \mathrm{~ns}$ as it is studied in [7].

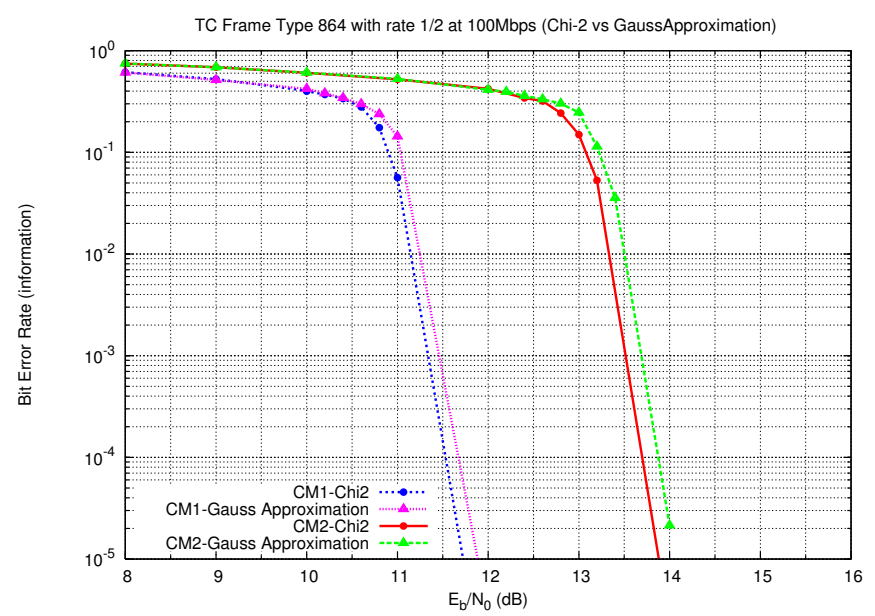

Fig. 3. Chi-squared vs Gaussian approximation in float precision for CM1 and CM2 using duo-binary turbo code at rate $1 / 2$ with $K=2$.

It is noticed that the results with Gaussian approximation match the chi-squared performances in floating point precision.

\section{B. Simulations in fixed point precision}

Simulation in fixed point precision is subject to hardware constraints. The duo-binary turbo coder hardware implementation is out of the scope of this paper. The digital design of the channel coder is furnished by TurboConcept for an optimum efficiency [20]. The energy detector of UWB platform is a logarithmic one [21]. To guarantee the scalar value of the energy $\mathcal{E}_{n, m}$ for equalization, a look-up table of the function $10^{x}$ is required. Computer simulations in fixed point precision are achieved by means of the SystemC class sc_fix [22]. The Gaussian approximation for energy equalization are computed through the look-up tables of the functions: $\phi(x)=\frac{e^{-x^{2} / 2}}{\sqrt{2 \pi}}, g(x)=1 / \sqrt{x}$ and $h(x)=10^{x}$. Figure 4 shows the Gaussian approximation computation architecture for the chi-squared distribution. According to the class $s c_{-} f i x$ of SystemC, a signed or an unsigned object are defined by two parameters: the total word length noted as $w l$, i.e. the total number of bits used in the type, and the integer word length noted as $i w l$, 


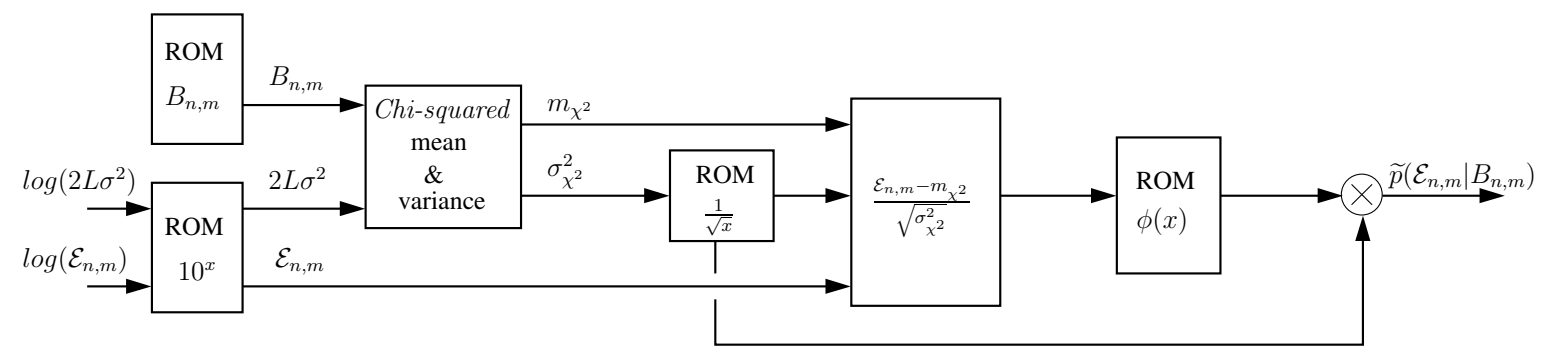

Fig. 4. Gaussian approximation architecture to the chi-squared distribution.

i.e. the number of bits that are on the left of the binary point (.) in a fixed point number. The remaining bits stand for the fractional part of the object. Hence each object is represented by a pair of parameters noted $\langle w l, i w l\rangle$. Simulations have been carried out with different parameter sizes. Table I shows the word sizes of the parameters considered for the fixed point simulations. We notice, that

TABLE I

PARAMETERS SIZE DEFINITION

\begin{tabular}{|c||c|}
\hline Parameters & Quantization size $<w l, i w l>$ \\
\hline \hline $\log \mathcal{E}_{n, m}$ & $<6,2>$ \\
\hline $\mathcal{E}_{n, m}$ & $<14,7>$ \\
\hline$B_{n, m}$ & $<6,2>$ \\
\hline$\sigma^{2}$ & $<6,1>$ \\
\hline$\widetilde{p}\left(\mathcal{E}_{n, m} \mid B_{n, m}\right)$ & $<7,1>$ \\
\hline$p\left(\mathscr{E}_{n} \mid x_{n}\right)$ & $<8,1>$ \\
\hline$\pi\left(x_{k}\right)$ & $<4,1>$ \\
\hline
\end{tabular}

the quantization error of the energy distribution is about $1 / 2^{7}$, which is bigger that the considered maximum error $\epsilon=5 \times 10^{-3}$. Then the quantization error will mask the error brought by the approximation of the chi-squared distribution.

Table II lists the Input/Output size look-up table necessary for density computation.

TABLE II

LOOK-UP TABLE InPut/OutPut SIZE

\begin{tabular}{|c||c|c|c|}
\hline Parameters & Input size & Output size & Table size (Kbits) \\
\hline \hline$\phi(x)=\frac{e^{-x^{2} / 2}}{\sqrt{2 \pi}}$ & $<8,2>$ & $<18,0>$ & 4.5 \\
\hline$g(x)=1 / \sqrt{x}$ & $<14,7>$ & $<10,4>$ & 160 \\
\hline$h(x)=10^{x}$ & $<6,2>$ & $<14,7>$ & 0.875 \\
\hline
\end{tabular}

We notice that the total memory occupied by the lookup tables is lower than the chi-squared look-up table considered previously with respect to $\mathcal{E}_{n, m}, B_{n, m}$ and $\sigma^{2}$. Although the simplifications defined in table II have led to reduce the amount of occupied memory, the inverse square root defined by $g(x)$ could be determined iteratively by applying the Newton-Raphson method [23]. The inverse square root of a parameter $a$ is obtained by the conver- gence of the suite (see Appendix I for details):

$$
u_{n+1}=\frac{1}{2} u_{n}\left(3-a u_{n}^{2}\right)
$$

where

$$
\lim _{x \rightarrow+\infty} u_{n}=\frac{1}{\sqrt{a}}
$$

The choice of the initial value $u_{0}$ is very critical for the convergence of (15) in a minimum of iterations.

Due to the difficulties to get an optimal generic initial value $u_{0}$ for a minimum iteration in hardware implementation, in what follows Table II is implemented instead of Newton-Raphson method.

Simulations according to table I and II under the same conditions as for double precision lead to the results depicted in Figure 5.

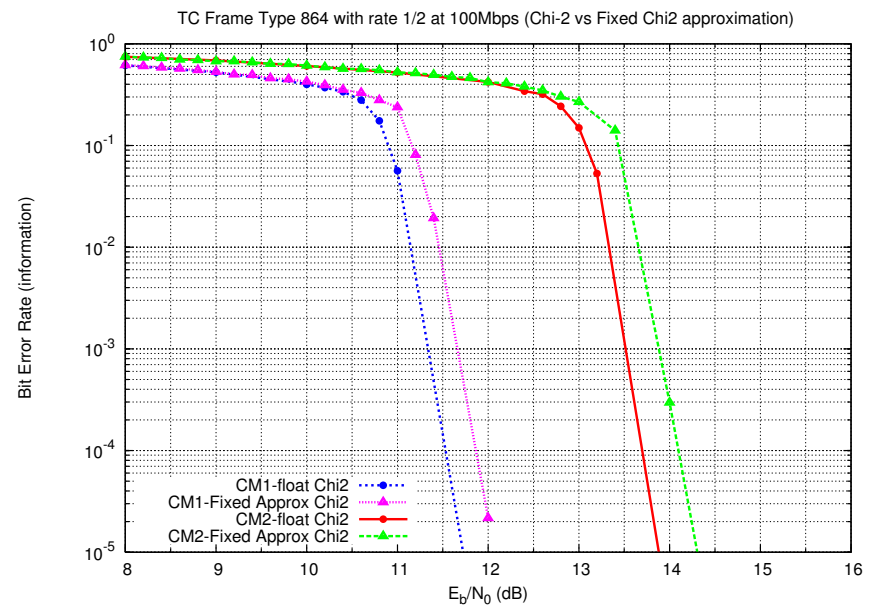

Fig. 5. chi-squared float precision versus the Gaussian approximation in fixed point precision for $K=2$.

Results in fixed point precision data types are close to those obtained in double precision with chi-squared distribution. These results are promising for a hardware implementation with the given parameters size. 


\section{Conclusion}

One purpose of this paper is to approximate the chisquared distribution by a gaussian distribution with mean and variance as the chi-squared to facilitate the hardware implementation in IR-UWB energy detection system. It is shown that the error is marginal and offers the way to hardware simplification. By using different parameter quantizations, it has been proven that the Gaussian approximation can largely reduce the necessary memory and the whole complexity of the probabilistic energy equalizer, with very limited performance decrease.

Further studies will be investigated for memory and processing optimization. For instance computing of probabilities could be done in logarithmic domain, or a polynomial approximation could be applied to replace some look-up tables. More research should be investigated on the initial value of Newton-Raphson method for digital design of the inverse square root, as it was studied in floating point precision [24].

\section{ApPENDIX I \\ Newton-Raphon Method}

Newton-Raphson method, also known as Newton's method, consists on computing the root that leads to $f(x)=0$ by the mean of iterative process, where $f(x)$ is a differentiable function defined on the interval $[a, b]$ with values in the real numbers $\mathbb{R}$. The following suite

$$
u_{n+1}=u_{n}-\frac{f\left(u_{n}\right)}{f^{\prime}\left(u_{n}\right)}
$$

converges to the root. The process is initialized with an arbitrary value $u_{0}$ that is important for a fast convergence (see [23], [25] for convergence proof and for more details). This procedure can be unstable near a horizontal asymptote or a local extremum. However, with a good initial choice of the root's position, the algorithm can be applied iteratively

\section{Newton-Raphson application to $g(x)=1 / \sqrt{x}$ :}

The function $g(x)$ is defined on the interval $] 0,+\infty[$ with value on $] 0,+\infty[$. Let $\alpha \in] 0,+\infty[$ such as $y=1 / \sqrt{\alpha}$, we define the function $f(x)$ by

$$
f(x)=\alpha+\frac{1}{x^{2}}
$$

where $f(x)$ is differentiable on the interval $] 0,+\infty[$ with value on $\mathbb{R}_{+}^{*}$. It is noticed that $1 / \sqrt{\alpha}$ is a root of $f(x)=0$. We note $u_{n}$ an approximation of the current root. A better approximation $u_{n+1}$ can be derived from (17) as

$$
u_{n+1}=\frac{1}{2} u_{n}\left(3-\alpha u_{n}^{2}\right)
$$

\section{REFERENCES}

[1] L. Yang and G. B. Giannakis, "Ultra-wideband communications: an idea whose time has come," IEEE Signal Processing Magazine, vol. 21, no. 6, pp. 26-54, Nov. 2004.
[2] J. D. Choi and W. E. Stark, "Performance of ultra-wideband communications with suboptimal receivers in multipath channels," IEEE Journal on Selected Areas in Communications, vol. 20, no. 9, pp. 1754-1766, DECEMBER 2002.

[3] R. Hoctor and H. Tomlinson, "Delay-hopped transmittedreference rf communications," IEEE Conference on UltraWideband Systems and Technologies, pp. 265 - 269, May 2002.

[4] V. Lottici, L. Wu, and Z. Tian, "Inter-symbol interference mitigation in high-data-rate uwb systems," IEEE International Conference on Communications, pp. 4299-4304, June 2007.

[5] Y. Zhang, H. Wu, Q. Zhang, and P. Zhang, "Interference mitigation for coexistence of heterogeneous ultra-wideband systems," EURASIP Journal on Wireless Communications and Networking, pp. 1-13, 2006.

[6] M. E. Şahin and H. Arslan, "Inter-symbol interfrence in high data rate uwb communications using energy detector receivers," IEEE International Conference on UWB, ICU, pp. 176-179, September 2005.

[7] S. Mekki, J. L. Danger, B. Miscopein, J. Schwoerer, and J. J. Boutros, "Probabilistic equalizer for ultra-wideband energy detection," IEEE 67th Vehicular Technology Conference (VTC), pp. 1108-1112, May 2008.

[8] J. G. Proakis, Digital Communications, 2nd ed. New York: McGraw Hill, 1989.

[9] N. C. Severo and M. Zelen, "Normal approximation to the chisquared and non-central $F$ probability functions," Biometrika, vol. 47, no. 3/4, pp. 411-416, December 1960.

[10] L. Canal, "A normal approximation for the chi-square distribution," Computational Statistics $\&$ Data Analysis, vol. 48, no. 4, pp. 803-808, April 2005.

[11] J.-T. Zhang, "Approximate and asymptotic distributions of chi-squared-type mixtures with applications," Journal of the American Statistical Association, vol. 100, pp. 273-285, March 2005.

[12] J. Foerster, "Channel modeling sub-committee report final," IEEE P802.15-02/368r5-SG3a, Tech. Rep., 18 November 2002.

[13] H. Urkowitz, "Energy detection of unknown deterministic signals," Proceedings of the IEEE, vol. 55, no. 4, pp. 523-531, April 1967.

[14] M. Abramowitz and I. A. Stegun, Handbook of Mathematical Functions with Formulas, Graphs, and Mathematical Tables, December 1972.

[15] A. H. M. Ross, "Algorithm for calculating the noncentral chisquare distribution," IEEE Transactions on Information Theory, vol. 45, no. 4, pp. 1327-1333, May 1999.

[16] R. Saadane, D. Aboutajdine, A. M. Hayar, and R. Knopp, "On the estimation of the degrees of freedom of in-door UWB channel," IEEE 61st, Vehicular Technology Conference (VTC), vol. 5, pp. 3147 - 3151, May 2005.

[17] S. Mekki, J. L. Danger, B. Miscopein, and J. J. Boutros, "Em channel estimation in a low-cost uwb receiver based on energy detection," Accepted in IEEE International Symposium on Wireless Communication Systems 2008 (ISWCS 08), 2008. [Online]. Available: http://comelec.enst.fr/ ${ }^{\text {mekki }}$

[18] "Digital Video Broadcasting (DVB); Interaction channel for Satellite Distribution Systems," ETSI EN 301790 V.1.3.1, Tech. Rep., March 2004.

[19] "Digital video broadcasting (DVB); interaction channel for satellite distribution systems; guidelines for the use of en 301 790," ETSI TR 101790 V.1.2.1, Tech. Rep., January 2003.

[20] "TC1000-xX DVB-RCS Turbo Decoder v2.1," TurboConcept, Tech. Rep., February 2005.

[21] AD8318 $1 \mathrm{MHz}$ to $8 \mathrm{GHz}, \quad 70 \mathrm{~dB}$ Logarithmic Detector/Controller. [Online]. Available: http://www.analog. com/en/prod $/ 0 \% 2 \mathrm{C} 2877 \% 2 \mathrm{CAD} 8318 \% 2 \mathrm{C} 00 . \mathrm{html}$

[22] SystemC User's Guide, version 2.0. [Online]. Available: http://www.systemc.org

[23] J.-M. Muller, Arithmétique des ordinateurs. Masson, 1989.

[24] C. Lomont, "Fast inverse square root," February 2003. [Online]. Available: http://www.lomont.org/Math/Papers/ 2003/InvSqrt.pdf

[25] C. Jordan, Calculus of Finite Differences, 2nd ed. CHELSEA PUBLISHING COMPANY, 1950. 